\title{
ESTUDIO DEL EMPLEO DE LOS PRONOMBRES CLÍTICOS $L E$ / LES CON REFERENTE PLURAL EN UN CORPUS ESCRITO DE LA REGIÓN NEA DE ARGENTINA
}

\author{
Study of the clitic pronouns le / les use with plural referent in a written corpus from \\ the Argentina NEA region
}

\author{
Hugo Roberto Wingeyer* \\ Universidad Nacional del Nordeste \\ hugowingeyer@gmail.com
}

\author{
Alejandro Angelina** \\ Universidad Nacional del Nordeste \\ alejandro.angelina@comunidad.unne.edu.ar
}

\begin{abstract}
Palabras clave el español de la región NEA de Argentina; pronombres clíticos; invariabilidad le por les; predicados triádicos
\end{abstract}

\section{Keywords}

Spanish of the Argentina NEA region; clitic pronouns; invariability le for les; triadic predicates

\section{RESUMEN}

Esta investigación tiene por objeto estudiar el uso de los pronombres clíticos le / les con referente plural de rasgo [+humano], en un corpus escrito de estudiantes universitarios de la región NEA de Argentina. Dado que la invariabilidad "le por les" (frecuente en muchas áreas hispánicas) es un fenómeno tan intenso y general en el área en estudio que nos permite formular la hipótesis de la proximidad a un cambio, resulta particularmente interesante conocer las estructuras y las posiciones que posibilitan la elección de uno u otro pronombre con referente plural. Para eso, abordamos el uso de los pronombres clíticos que se encuentran regidos por tres predicados triádicos - "dar", salir" y "otorgar"- que en los contextos lingüísticos analizados restringen el uso de clíticos de acusativo, dando lugar solo a los de dativo, que son las unidades consideradas en este trabajo.

\section{Abstract}

The aim of this research is to study the use of the clitic pronouns le / les with plural referent of [+human] feature, in a written corpus of university students from the Argentina NEA region. Given that the invariability "le for les" (frequent in many Hispanic areas) is such an intense and general phenomenon in the area under study that we can hypothesize the proximity to a change, it is particularly interesting to know the structures and positions that make possible the choice of one or the other pronoun with plural referent. To this end, we address the use of clitic pronouns which are governed by three triadic predicates - "dar", "salir" and "otorgar"- which in the linguistic contexts analysed restrict the use of accusative clitics, giving way only to dative clitics, which are the units considered in this paper. 


\section{Introducción}

El presente trabajo tiene como objeto de estudio el uso de los pronombres átonos (clíticos) "le / les" con referente plural de rasgo [+humano], en producciones escritas de estudiantes de la Facultad de Humanidades de la Universidad Nacional del Nordeste (UNNE). Las muestras recogidas para la confección del corpus, que datan de 2018, son producciones textuales de secuencia explicativoargumentativa, enmarcadas en las actividades de una materia de primer año, el Taller de Comprensión y Producción de Textos del Departamento de Letras.

Dado que nuestra comunidad es distinguidora de caso, tal como lo demuestran los trabajos publicados por Abadía de Quant $(2003$,2004), resulta de interés identificar las estructuras y posiciones que posibilitan la elección de uno u otro pronombre con referente plural de rasgo [+humano]. Para lograrlo, abordamos el uso de estos clíticos regidos por tres predicados triádicos: "dar", "salir" y "otorgar".

Las preguntas que orientan nuestro estudio son las siguientes: a. en qué medida se mantiene el uso de "le" por "les" con referente plural de rasgo [+humano] en nuestra comunidad de habla; b. qué implicancias podría tener el acercamiento o la posición de ese tipo de referente en relación con el clítico seleccionado; y c. qué líneas reflexivas podemos trazar a partir de nuestros resultados, en relación con los obtenidos por Abadía de Quant.

\section{Marco teórico}

\subsection{Invariabilidad: le por les. Consideraciones generales}

El uso del clítico de dativo singular con referencia plural, de uso frecuente en el español de España, tanto antiguo como moderno, y muy común en toda América (Kany, 1994; Fontanella de Weinberg, 1993; Company, 2006), implica su despronominalización: "la pérdida de sus capacidades anafóricas o deícticas y, por lo tanto, su progresiva conversión en una marca de función sintáctica o afijo verbal, que anticipa que en la cláusula en la que se encuentra existe otro argumento" (Gómez Seibane, 2012, p. 71). En este sentido, la pérdida de la concordancia plural del clítico de objeto indirecto pudo haber sido propiciada por la ambigüedad de determinados contextos acerca del número, en los que la localización del referente del pronombre personal era confusa (Company, 2006, p. 543-549).

Por su parte, desde otra perspectiva, García Negroni (2010,p. 426) considera que la discordancia en estudio, de uso frecuente tanto en España como en América, "se da incluso en hablantes cultos" y que es "normativamente desaconsejable" en lengua escrita.

\subsection{Invariabilidad: le por les en el español de la región NEA de Argentina}

En su caracterización sincrónica del español del nordeste argentino, Abadía de Quant (2004) presenta el uso del pronombre de objeto directo le con valor singular y plural, como "un problema 
de concordancia sintáctica de independencia absoluta con la elisión de /-s/", ${ }^{1}$ de "uso extendido en todos los isolectos" (p. 113). Lo incluye en un conjunto de fenómenos que "siendo comunes a otras áreas hispánicas, se presentan en la zona con un rasgo de generalidad e intensidad tales que permiten la hipótesis de la proximidad a un cambio" (p. 109-110).

En su dimensión diacrónica, el uso del clítico de dativo singular con referencia plural es tratado por Abadía de Quant (2003) en el eje dedicado a fenómenos lingüísticos del nivel morfosintáctico del español de procedencia medieval. Del origen de esta variante ("le" por "les"), adhiere a la hipótesis que presentan Keniston (1937) y Kany (1994): el uso de la forma singular en detrimento de su correlato plural "les" se debe a la equiparación de formas que derivan de las combinaciones registradas en el español medieval "ge lo" "se lo", en donde el pronombre dativo ge o se es usado tanto para plural como para singular, como variante alomórfica de "le / les", "que lo sustituye cuando va seguido por un clítico acusativo: Juan se lo vendió, y no Juan le lo vendió", tal como ilustra Di Tullio (2005, p. 126). Así, para Abadía de Quant "el hablante extiende a le, las características de singular y plural propias de ge-se" (2003, p.128); variante sintáctica que la autora registra "con frecuencia" desde mediados del siglo XVI hasta fines del siglo XIX en el español documentado de Corrientes. Dos de los ejemplos que nos presenta:

(a) sin reparar en el daño que rreciben nyenel engaño que se le haze ( $A C E^{2}$ 6-9-1569).

(b) me encargas le participe a Genaro y tu tía Rosa quienes con tus servidores los felicita ( $C P$ 14-12-1865).

Como podemos observar, ambos clíticos poseen referente persona en plural. En (a) suponemos esto por el rasgo [+humano] del predicado "engañar". Por otra parte, no comparten igual posición dado que en (a) ocupa posición anafórica -con elisión de referente pero codificado en el rasgo morfológico de 3ra persona de plural en "reciben"- y en (b) "le" antecede al referente compuesto "a Genaro y su tía Rosa".

Volviendo a la dimensión diacrónica, hacia principios de este siglo, el uso de "le" con valor plural se mantiene en el español sincrónico de Corrientes, "se registra ampliamente extendido" y en situaciones sintácticas muy variadas, en oposición a lo planteado por la gramática oficial del español, para la que su uso es “ocasional” (Abadía de Quant, 2003, p. 129).

Recuperamos a continuación los ejemplos recopilados por Abadía de Quant en 2003 (p. 130). A los fines que perseguimos en este artículo es importante mencionar que los entornos sintácticos de los ejemplos son variados, que los referentes obedecen al rasgo [+humano], y que la posición del clítico dativo en relación con su referente puede ser anafórica como en (d), (e) y (g), o bien catafórica como en (c) y (f):

1. De acuerdo con Abadía de Quant (2004), la elisión de /-s/ es un "rasgo tradicional en la zona”, que seguramente fue "reforzado por el guaraní que no tiene en su sistema /-s/" (p.106).

2. Actas Capitulares Editadas -en (a) - y Correspondencia Particular -en (b)-, del Archivo Histórico de la Provincia de Corrientes. Luego de las siglas se lee la fecha de origen del documento. Las demás fuentes escritas que utiliza la autora son las siguientes: Actas Capitulares, Correspondencia Oficial, Documentos de Gobierno, Documentos Oficiales, Protocolos, Documentos incluidos en Fondo Mantilla y Judiciales (Quant, 2003). 
(c) Yo ya le avisé pronto (inten.) ${ }^{3}$ a lo que viven por aquí de que esos son chorro. (ss) ${ }^{4}$

(d) ... y sola la madre le dio (a sus hijos) toda la educación, eso sí que sola... sin hombre. (ss)

(e) -Pero usted me dijo que ella atiende bien a los hijos...

-Sí pue, atiende... pero no le cocina como debe ser... guiso eso. (ss)

(f) -Ustedes van a hacer alianza con otros partidos ¿’o van solos (a las elecciones)?

-Nosotros queremos que la democracia se afiance y le vamos a ofrecer posibilidades a todos.

Ellos van a decidir. $(\mathrm{s})^{5}$

(g) A los pobres malloneros perjudican porque le sacan todo y venden el dorado en Santa Fe, Buenos Aires. (s).

De los ejemplos (a) a (g) se pueden rescatar algunas regularidades en el comportamiento de los predicados que rigen dativo, lo que implica el uso de una u otra variante, "le" o "les":

1. todos los referentes de estos pronombres átonos distinguen plural e implican personas o conjunto de estas;

2. desde la perspectiva gramatical descriptiva de Di Tullio (2005) y en vinculación con la estructura argumental de los verbos (los que activan el uso de las variantes átonas), son verbos de acción que tienen un "dativo benefactivo" que "denota la entidad beneficiada (o perjudicada) por la acción” (p. 128): en casos del español diacrónico se documenta el engaño que se le hace y le participe a Genaro y su tía Rosa, en (a) y (b) respectivamente; a la actualidad corresponden casos como no le cocina como debe ser y le sacan todo, en (e) y (g); y

3. la identificación de estos usos dan cuenta de dos variables que atender a la hora de estudiar este fenómeno según Aleza (2013): la cercanía o no del clítico respecto de su referente plural (por ejemplo, si comparten oración o no) y la posición anafórica o catafórica respecto de aquel. Así, para esta autora la "inmovilización del pronombre le con referencia plural" se debería a que el pronombre está afectado por un proceso de gramaticalización con pérdida de la referencia deíctica "para funcionar como un marcador de la existencia de un elemento de complemento indirecto en la oración” (p. 21).

Sobre la base de estos aportes teóricos presentamos a continuación la metodología aplicada al tratamiento de nuestro corpus para su posterior análisis.

\footnotetext{
3. "Pronto" (no con valor de "rápidamente") en ese caso es una partícula intensificadora: "enfatizador que se pospone a la palabra que se desea resaltar. Se enfatizan verbos, sustantivos, pronombres y adverbios" (Abadía de Quant, 2003, 237). Es un rasgo sintáctico-semántico del español sincrónico de Corrientes producto de la identificación que los hablantes han hecho con "voi", que también funciona como adverbio de tiempo al igual que nuestro "pronto". Obsérvese el par (a) Vino pronto a su casa / (b) Tarde pronto te arrepentiste (reformulado de Abadia de Quant, 2003): solo en (b) funciona como intensificador.

4. Nivel subestándar: refiere a hablantes "varones y mujeres sin escolaridad (o que desertaron muy tempranamente el sistema)" (Abadía de Quant, 2003, p. 4).

5. Nivel estándar: conformado por hablantes "varones y mujeres con escolaridad primaria completa, estudios secundarios completos, estudios terciarios completos o incompletos" (Abadía de Quant, 2003, p. 4).
} 


\section{Metodología y formación del corpus}

El corpus está formado por las producciones escritas de estudiantes de primer año de las carreras Profesorado y Licenciatura en Letras, y Profesorado y Licenciatura en Ciencias de la Educación, en el marco de la cátedra Taller de Comprensión y Producción de Textos del Departamento de Letras, de la Facultad de Humanidades de la Universidad Nacional del Nordeste (UNNE). Se trata de 56 muestras recogidas en 2018; son producciones textuales de secuencia explicativo-argumentativa, corolario de un trabajo práctico escrito domiciliario.

En esta oportunidad presentamos 15 de 56 secuencias enunciativas en las que los pronombres átonos desempeñan función de objeto indirecto. Cada secuencia enunciativa corresponde a un estudiante. Los trabajos fueron entregados la última semana de clases del primer cuatrimestre del año 2018. Los pasajes escritos sobre los que hicimos foco son dos, los que responden a las inquietudes que se desarrollan en el texto fuente:

a. qué espera la sociedad de los intelectuales y por qué la autora considera su respuesta como una fantasía; y

b. si los intelectuales logran o no realizar su tarea en la sociedad.

Los signos auxiliares utilizados para el tratamiento de los casos tienen el significado que especificamos a continuación. Cabe aclarar que realizamos mínimas modificaciones que pudieran ayudar a la comprensión, respetando lo máximo posible las producciones originales: 6

[ ] reposición de una unidad lingüística o signo de puntuación

(...) recorte del enunciado fuente

le $\sim$ les clíticos de dativo

$\underline{\text { ellos }}$ los intelectuales referente plural [+humano] de clíticos dativos

cursiva verbos no que rigen clíticos dativos

\section{Análisis del corpus}

Para empezar, abordamos en nuestro análisis del corpus el uso de los pronombres personales de tercera persona que se encuentran regidos por tres predicados triádicos: "dar", "salir" y "otorgar", que en los contextos lingüísticos analizados restringen el uso de clíticos de acusativo, dando lugar solo a los de dativo, unidades estimadas en este trabajo.

Nuestras preguntas orientadoras, que surgen del objeto de estudio, pueden formularse del siguiente modo: a. en qué medida se mantiene el uso de "le" por "les" con referente plural de rasgo

6. A cambio, exponemos estructuras oracionales prototípicas para ilustrar fenómenos más complejos, vale decir, aquellos extraídos de las producciones escritas de estudiantes. 
[+humano] en nuestra comunidad de habla; b. qué implicancias podría tener el acercamiento o la posición de ese tipo de referente en relación con el clítico seleccionado; y c. qué líneas reflexivas podemos trazar a partir de nuestros resultados, en relación con los obtenidos por Abadía de Quant.

Luego, advertimos que los predicados verbales triádicos utilizados por los estudiantes son "dar", "otorgar" y -con un solo uso- "imprimir". Las consecuencias de esta naturaleza triádica se manifiestan en la estructura sintáctica de las secuencias que analizamos. Presentamos las siguientes representaciones formales de las formulaciones de enunciados de nuestro corpus, a modo ilustrativo ${ }^{7}$ (en los tres casos estabilizamos solo las estructuras, como se verá, no el número de argumentos que selecciona cada predicado):
(A) Los intelectuales(les) dan un sentido a la vida.
(B) Los intelectuales(les) otorgan un valor a la realidad.
(C) Ellos(les) imprimen un sentido a los fenómenos.

Semánticamente, y con implicancias en el número de argumentos que seleccionan, estos verbos tienen la propiedad de ser ditransitivos, es decir, "seleccionan un OD y un OI” (Di Tullio, 2005, p. 127).

Los demás casos están sintetizados a continuación en (D). Presentan el uso de la locución verbal "salirle algo a alguien" reactualizada como triádica en nuestro corpus, como los predicados de (A), (B) y (C) pero no ditransitiva, sino inacusativa: al igual que "gustar", "encantar", "interesar", con sujeto pospuesto, decir la verdad en este caso.

\section{(D) A ellos les sale decir la verdad (a la gente)}

En cuanto al comportamiento del clítico dativo en casos como (A), (B) y (C) funciona en coaparición con su referente, aunque el pronombre admita elisión con verbos ditransitivos (de frecuencia escasa ${ }^{8}$ en los casos identificados por nosotros): Los intelectuales() dan un sentido a la vida, Ellos () otorgan un valor a la realidad, Ellos () imprimen un sentido a los fenómenos. En (D), en cambio, la presencia de "les" -o su variante en singular- no puede elidirse (o al menos no lo hacen nuestros estudiantes en estas estructuras). ${ }^{9}$

Finalmente, luego de una rutina exploratoria podemos afirmar que de 56 producciones escritas -una por cada estudiante- solo 17 no registran usos de clíticos de dativo debido a la naturaleza de los predicados que cada estudiante utiliza en los pasajes de secuencia explicativa, los que no posibilitan la relación entre el pronombre "les" (o su variante "le") y su referente en plural de rasgo [+humano].

A modo conjetural, decimos que esto sucede porque, entre otros factores, los verbos que marcamos en cursiva son diádicos (Di Tullio, 2005, p. 127), vale decir, seleccionan dos construcciones

7. Los predicados verbales están en negrita; los tres argumentos, en cursiva. Como oportunamente analizamos, la presencia de "(les)" en estas oraciones es obligatoria en algunos casos, en otros no.

8. Es lo que sucede con "pedir", en el caso de nuestro corpus, ver (3:) La ensayista Beatriz no tiene muy bien definido qué es lo que la sociedad pide o espera de los intelectuales [.] certifica que su opinión es una fantasía (...).

9. Referimos a casos del tipo *A ellos no () sale decir la verdad. 
para completar un enunciado (diferente de predicados triádicos, que facilitan la presencia de clíticos de dativo). A modo de ilustración y a través de una reducción sintáctica, vemos esto en esquemas como " $\mathrm{x}$ cumple (con) y", "x llega a concretar y", etc. Es lo que sucede con estos tres casos ilustrativos de nuestro corpus:

(1) Muchas veces los intelectuales no cumplen su tarea de crear [un] sentido. Son muy pocos los que llegan a concretar dicha tarea.

(2) Beatriz también menciona que muy pocos son los que cumplen esa tarea y se ven iluminados. Esto es acertado, considerando que la sociedad donde [los intelectuales] ejercen sus discursos no lo[s] aceptan y el trabajo de los intelectuales se afecta (...).

(3) La ensayista Beatriz no tiene muy bien definido qué es lo que la sociedad pide o espera de los intelectuales, certifica que su opinión es una fantasía (...).

Los 39 estudiantes restantes presentan usos de los clíticos dativos con referencia plural de persona. Los clasificamos según tres formas de aparición o uso de estas unidades:

4.1 usos de "le" con referente en plural: 13 estudiantes;

4.2 uso de "les" con referente en plural: 11 estudiantes; $y$

4.3 uso de "le" y "les" que comparten referente en plural: 15 estudiantes.

Para los dos primeros grupos seleccionamos cinco casos; para el último, dos.

\subsection{Usos del pronombre clítico "le" con referente plural}

(4) (...) a modo de respuesta [Sarlo] especula una fantasía [:] que los intelectuales le den sentido a un acontecimiento y que esa idea pueda subsistir en la sociedad.

(5) Los intelectuales son aquellos que tienen que darle ese sentido, pero no siempre le sale y a veces ni $\operatorname{tratan}(\ldots)$.

(6) A los intelectuales no siempre le sale su tarea porque no a todos le interesa lo que se está diciendo, no todos interpretan igual (...).

(7) Los intelectuales le dan un sentido a lo bueno y a lo malo, por eso ella [Sarlo] considera que su respuesta es una fantasía (...) Según la entrevistada no siempre le sale su tarea a los intelectuales.

(8) Creo que muchas veces no le sale a los intelectuales su tarea porque como anteriormente escribí, no transmiten un tema que dé sentido a la sociedad.

En todos estos casos, el pronombre dativo y su referente (o su correspondiente relevo léxico con pronombre demostrativo en plural) comparten oración. Además, aunque encontramos usos de clítico en posición catafórica -como en el segundo uso en (7) y en (8)-, son más frecuentes los casos en posición anafórica -en (4), (5), (6) y el primer uso en (7)-.

\subsection{Usos de pronombre clítico "les" con referente en plural}

(9) En mi opinión, no siempre les sale su tarea porque es muy difícil encontrar un tema que interese y represente a la gente [.] no logran llegar a ella debido a que los intelectuales hablan o estudian un tema de interés propio de su área. 
(10) Entonces, esta necesidad de sentido es antropológica y se reproduce en las sociedades modernas, y los intelectuales serían aquellos que deberían tratar de dar un sentido. Sin embargo, no siempre les sale dar un sentido o más bien no siempre tratan.

(11) La entrevistada asegura que lo que la sociedad espera de los intelectuales es que les den un sentido a los acontecimientos sociales, políticos, culturales, etc. (...) A los intelectuales no siempre les sale su tarea porque que una persona sea intelectual no quiere decir que sepa todo de todos los temas.

(12) (...) hay sucesos que no pueden ser explicados. Y a veces eso es la causa de que a $\underline{\text { los }}$ intelectuales no les salga ser intelectuales.

(13) Entonces por esta fantasía, es que la sociedad pide a los intelectuales que se encarguen de estos significados, pero no siempre les sale bien [.] como lo indica Sarlo, están o no iluminados para que les salga bien.

El enunciado (9) inicia con una construcción modalizante, con lo cual no hay referente antepuesto al clítico. Todos los demás casos son de carácter anafórico y comparten oración con el referente. El segundo caso de (13) es especial por estar en posición posterior a un "les" antepuesto y en oración aparte. ${ }^{10}$

\subsection{Usos de pronombres "le" y "les" que comparten referente en plural}

(14) Por otro lado, ella [Sarlo] sí tiene una fantasía personal, pensar que ellos le otorgan un sentido a la realidad, en términos antropológicos, científicos y filosóficos. Piensa que ellos deberían, si les sale, imprimirle un sentido a los fenómenos, aunque no siempre lo consiguen.

(15) Yo creo que no siempre le sale a los intelectuales su tarea de tratar de dar un sentido; porque como dice la ensayista, no siempre les sale y no siempre tratan porque no están iluminados.

En (14) observamos el uso de ambos clíticos que comparten oración con sus referentes. Es interesante el comportamiento de "les" en la construcción aclaratoria "si les sale", la que por no compartir oración con el referente "los intelectuales", vendría a remarcar los rasgos de persona, plural; algo que quizá no se presenta como necesario en los usos de "le" (creemos, gramaticalizado) con "otorgar" e "imprimir" por la cercanía con sus sujetos ("ellos").

En (15) encontramos un uso catafórico del clítico en singular regido junto con su referente por el verbo "salir". La presencia del uso anafórico de "les" estaría evidenciando la necesidad por parte de quien escribe de recuperar, a través de la concordancia del clítico, los rasgos del referente expreso en la oración anterior, y no recuperado en la última.

\section{A modo de cierre}

Sobre la base del análisis del corpus, respondemos las preguntas formuladas en esta investigación:

a. Respecto de en qué medida se mantiene el uso de "le" por "les" con referente plural de rasgo [+humano] en nuestra comunidad de habla, comprobamos que en 15 casos de 39 analizados presentan

10. El punto y seguido (aparece borrado en el original) fue colocado por nosotros. 
usos de "le" y "les" dentro de una misma oración o en oraciones distintas, tanto en posición anafórica como catafórica. Esto sucede con verbos ditransitivos donde bien podría no manifestarse uno u otro clítico. En casos con verbos inacusativos - de uso obligatorio- no observamos elisión de pronombre dativo. En este sentido, no podemos afirmar que la tendencia hacia un sincretismo formal entre "le / les” con referente plural [+humano] esté resuelta. Por el contrario, advertimos una inestabilidad en el uso de una y otra formas, incluso en casos donde ambas pueden compartir referente. Sí podemos decir, tal como arrojan los casos expuestos, que hay una inclinación marcada hacia el uso de "le" en los casos mencionados en detrimento de la forma tradicional y canónica "les".

b. En lo referido a qué implicancias podría tener el acercamiento o la posición de ese tipo de referente en relación con el clítico seleccionado, observamos que la mayor cantidad de casos procesados indica que los usos de los clíticos dativos tienen posición anafórica y comparten oración. Identificamos casos donde incluso hay usos dobles (con cambio o no de referente a través de pronombres personales y/o construcciones nominales equivalentes). En cuanto a los usos catafóricos, sería interesante analizarlos en escritos de redacción y tema libres para identificar con qué frecuencia se usan. Entendemos que los dativos catafóricos de nuestro corpus quizá se vean motivados por la presencia del referente expreso en las actividades que proponemos a estudiantes (nuestros informantes, en este caso).

c. Finalmente, en lo que atañe a la líneas reflexivas que podemos trazar a partir de nuestros resultados -en relación con los obtenidos por Abadía de Quant (2003,2004)-, tal como arroja nuestro análisis, se evidencia la pervivencia de la fluctuación en el uso de "le / les" con referente plural de rasgo [+humano] sin ninguna restricción de entorno sintáctico (Abadía de Quant, 2003, p.130) en hablantes de Resistencia y Corrientes, en el campo de la producción escrita académica. Cuando decimos hablantes nos referimos a estudiantes de la universidad con estudios primarios y secundarios completos. Una muestra que quizá podría ser catalogada, desde la perspectiva de los estudios de Abadía de Quant (2003, p.4), como pertenecientes a un nivel estándar.

\section{Referencias bibliográficas}

Abadía de Quant, I. (2003). Aspectos del español coloquial sincrónico de los nativos de la capital de Corrientes (Argentina). Resistencia, Universidad Nacional del Nordeste.

Abadía de Quant, I. (2004). El español del Nordeste. En Fontanella de Weinberg, M. (ed.), El español de la Argentina y sus variedades regionales (pp. 121-160). Bahía Blanca, Asociación Bernardino Rivadavia.

Aleza, M. (2013). Estudio del empleo de los pronombres clíticos en un corpus oral de La Habana (Cuba). Borealis: An International Journal of Hispanic Linguistics, 2 (1), 1-34. http://dx.doi. org/10.7557/1.2.1.2439

Company, C. (2006). El objeto indirecto. En Company, C. (dir.), Sintaxis histórica de la lengua española. Primera parte: La frase verbal (vol. 1, pp. 477-572). México, Fondo de Cultura Económica.

Di Tullio, A. (2005). Manual de gramática del español. Buenos Aires, Waldhuter. 
Fontanella de Weinberg, M. B. (1993). El español de América. Madrid, MAPFRE.

García Negroni, M. M. (2010). Escribir en español.Claves para una corrección de estilo. Buenos Aires, Santiago Arcos editor.

Gómez Seibane, S. (2012). Los pronombres átonos (le, la, lo) en el español. Madrid, Arco Libros.

Kany, Ch. E. (1994). Sintaxis hispanoamericana. Madrid, Gredos.

Keniston, H. (1937). The Syntax of Castilian Prose. The Sixteenth Century. Chicago, The University of Chicago Press.

*Hugo Roberto Wingeyer es Doctor por la Universidad de Alcalá (Programa de Lingüística Aplicada) y se desempeña como Profesor Titular de Historia del Español y del Taller de Comprensión y Producción de Textos de la Facultad (Universidad Nacional del Nordeste). Dirige el Proyecto de Investigación: "Descripción y análisis de prácticas letradas académicas de estudiantes de la Facultad de Humanidades de la UNNE 2017/2020” (SGCyT-UNNE).

**Alejandro Angelina es Profesor en Letras (Universidad Nacional del Nordeste) y alumno regular de la Maestría de Enseñanza de Español como Lengua Extranjera (Universidad Nacional de Córdoba). Es profesor Adjunto en las cátedras Taller de Comprensión y Producción de Textos y Lingüística II de las Carreras Licenciatura y Profesorado en Letras (Facultad de Humanidades, UNNE). Forma parte del Proyecto de Investigación "La adquisición del discurso académico escrito y oral en la enseñanza del español como lengua materna y extranjera. Distintos aspectos de un proceso de subjetivación en estudiantes de la UNNE" (SGCyT-UNNE).

RECIBIDO: $15 / 11 / 2020$

ACEPTADO: 15/12/2020 